\title{
El concepto de intersubjetividad de Niklas Luhmann
}

\author{
Sergio Pignuoli Ocampo* \\ Consejo Nacional de Investigaciones Científicas y Técnicas, \\ Universidad de Buenos Aires, Buenos Aires, Argentina
}

\section{RESUMEN}

En el presente trabajo reconstruimos el concepto de intersubjetividad delineado por Niklas Luhmann tras el 'giro comunicativo' que imprimió a su teoría general de sistemas sociales (TGSS) en los ańos 80; en pos de ampliarlo elaboramos sobre su base dos determinaciones ulteriores relativas a su vínculo con el fenómeno de la agregación y a su conexión con el proceso comunicativo básico. La hipótesis defendida es que Luhmann redefinió comunicativamente la intersubjetividad como formación de un tipo específico de estructuras, denominadas estructuras concordantes, moduladas como consenso o como disenso.

\section{Palabras clave}

Intersubjetividad, comunicación, consenso, disenso, agregación

\section{Niklas Luhmann's concept of intersubjectivity}

\section{ABSTRACT}

In this paper we reconstruct the concept of intersubjectivity as outlined by Niklas Luhmann following the 'communicative turn' he gave his General Social Systems Theory (GSST) in the Eighties. Based on this notion, we further expound on two

* Magíster en Comunicación y Cultura y licenciado en Sociología, Universidad de Buenos Aires (UBA). Actualmente es docente de la cátedra 'Niklas Luhmann y la sociología de la modernidad', Facultad de Ciencias Sociales de la mencionada casa de estudios (UBA-FCS) y becario doctoral del Consejo Nacional de Investigaciones Científicas y Técnicas (Conicet) con sede de investigación en el Centro Cultural de la Cooperación (CCC). Correo electrónico: spignuoli@conicet.gov.ar. Este trabajo cuenta con el apoyo financiero del proyecto de investigación "Análisis sistémico de las transformaciones de la unidad selectiva de la comunicación producidas por la convergencia tecnológica y las redes sociales en el contexto de la utilización de tecnologías digitalizadas y móviles de la información y de la comunicación" (UBACyT 20020110200047). 
subsequent affirmations relative to its link with the phenomenon of aggregation and its connection with the basic communications process. The hypothesis is that Luhmann communicatively redefined intersubjectivity as the formation of a specific type of structures, called concordant structures, modulated as a consensus or as dissent.

Keywords

Intersubjectivity, communication, consensus, dissensus, aggregation

\section{Introducción}

En este trabajo reconstruiremos el concepto de intersubjetividad elaborado por Niklas Luhmann a partir de la transformación del fundamento operativo que imprimió a su programa de investigación en los años 80 , y sobre la base de esta reconstrucción delinearemos luego dos determinaciones adicionales al concepto: la primera relativa al vínculo entre intersubjetividad y agregación en sistemas complejos; la segunda relativa a la conexión entre la formación de estructuras intersubjetivas y a la distinción entre temas, tematización y contribuciones.

Si bien es cierto que durante ese período el autor elaboró una crítica al concepto de intersubjetividad, cuyo eje era declararla impertinente para determinar el objeto sociológico, es preciso no apresurar conclusiones al respecto, pues Luhmann no sólo declaró la debilidad analítica de la intersubjetividad frente a la autopoiesis de la comunicación en lo referente a la unidad del objeto, sino que además propuso la reformulación de ella sobre premisas comunicativas. En esta redefinición el autor hizo eje en la formación de concordancia (Übereinstimmung) comunicativa en los sistemas sociales. ${ }^{1}$ Dado que esta reformulación ha sido mayormente desatendida, consideramos necesario reconstruirla, primero, para hacer justicia con la posición integral del autor, y, segundo, para establecer las premisas de una conceptualización sistémica de la intersubjetividad.

La reformulación comunicativa de la intersubjetividad, como mencionamos, no ha recibido aún la atención debida; sin embargo, no ha pasado totalmente inadvertida. Wolfgang Schneider (1994) señaló que desde la perspectiva de la teoría de sistemas la intersubjetividad no desaparece, sino que se convierte en una construcción comunicativa. Wendelin Reich (2010) indicó que la concep-

En este trabajo todo uso de la voz española concordancia estará referido a la voz alemana Übereinstimmung. Seguimos, en este sentido, la traducción empleada por Beriain y García Blanco en Luhmann (1998b). 
ción de estructura social como 'creación de redundancia' comunicativa conecta a Luhmann, a través de Bateson, con Garfinkel y Schegloff, quienes conciben la intersubjetividad como 'logro secuencial' (sequential achievement). Retomaremos, entonces, la reconstrucción del concepto avanzada por estos autores, y sobre su base delinearemos sus relaciones con otras zonas del programa sistémico, en especial con el concepto de estructura y con la distinción entre temas y aportes. De este modo, nos proponemos problematizar el concepto comunicativo de intersubjetividad propuesto por Luhmann. Nuestros interrogantes se ordenan en torno a tres preguntas básicas: ¿cómo se forma la intersubjetividad en los sistemas sociales?; ¡ cuál es su función comunicativa?; ¡con qué componentes de los sistemas sociales se vincula?

Nuestra hipótesis es que la intersubjetividad es un tipo de formación de estructuras de expectativas comunicativas caracterizadas por la concordancia. Esta dicha formación estructural puede modularse de manera positiva (consenso) o de manera negativa (disenso) e implica necesariamente un mecanismo intrasistémico de selección agregativa de expectativas. ${ }^{2}$ El rasgo distintivo de las estructuras concordantes de la intersubjetividad es organizar, dentro de la dimensión social de sentido de la comunicación, horizonte(s) de expectativas de baja heterogeneidad.

La hipótesis merece tres aclaraciones. En primer lugar, subrayamos que nuestra hipótesis sugiere una asociación primaria de la intersubjetividad con la formación de estructuras de los sistemas sociales, no con las improbabilidades de la comunicación; por consiguiente, la intersubjetividad no es un equivalente funcional de los medios de comunicación, ni de los orientados al entendimiento ni de los orientados a la consecución. En segundo lugar, la formación de la intersubjetividad sistémicamente concebida consiste en una diferenciación estructural únicamente posible dentro de la recursividad de la comunicación ni es un presupuesto tercero ni es ajeno a ella, por tanto es compatible con la clausura operativa del sistema social y portadora de todas las propiedades imputadas a las estructuras por la sociología sistémica de Luhmann: morfogénesis, estilización, plasticidad, reversibilidad, acoplabilidad, latencia. En tercer lugar, destacamos que la intersubjetividad comunicativa, así concebida, implica una desagregación

2 Cabe destacar que los conceptos de consenso y disenso no serán empleados aquí en su sentido corriente, sino en relación con los desarrollos teóricos de Niklas Luhmann. Según el autor, consenso y disenso conforman el doble horizonte de la dimensión social del sentido comunicativo. El consenso se caracteriza por modular un horizonte de expectativas favorable en torno a una tematización; en tanto que el disenso, por uno desfavorable. En el cuerpo central del trabajo enfocamos uno de los rasgos distintivos de la posición de Luhmann: ambos conceptos implican la formación de concordancia comunicativa. Esta no es privativa del consenso. En ello radica una de las características decisivas de la propuesta del autor, que procuramos proseguir. 
de la descripción sistémica de la autoorganización de los sistemas sociales, y no una contraposición con ella. El rasgo de la intersubjetividad que nos interesa poner de manifiesto es la propiedad agregativa de su mecanismo selectivo; de manera tal que, primero, recalcamos el carácter multinivelado o multiestratificado de la formación de estructuras comunicativas, y, segundo, ofrecemos una distinción adicional para la descripción de este nivel.

La elaboración de un corpus de trabajo para tratar esta cuestión no nos ha presentado dificultades mayores. Nos concentramos fundamentalmente en dos trabajos del autor: Sistemas sociales (Luhmann, 1998a) y el artículo "iIntersubjetividad o comunicación?” (Luhmann, 1998b). Para la discusión de la evolución incluimos los primeros tres volúmenes de Ilustración sociológica, aunque nos concentramos en el primero de ellos, pues este ofrece numerosos elementos sobre el tema que nos convoca (Luhmann, 1973).

En el desarrollo del cuerpo central del trabajo nos proponemos deslindar el concepto sistémico de intersubjetividad y conectarlo con el sistema de categorías de la comunicación. El plan expositivo es el siguiente: sintetizaremos la evolución del concepto de intersubjetividad en Luhmann, dejando así constancia del interés del autor por tal tema (2), luego reconstruiremos el concepto comunicativo de intersubjetividad (3), tras ello trataremos el concepto de agregación (4), para después vincularlo con la distinción entre temas y contribuciones (5). Finalmente, presentaremos los resultados y discutiremos las conclusiones (6).

\section{La evolución del concepto de intersubjetividad en Luhmann}

Ni repentino en los años 80 ni exclusivamente crítico, el interés de Luhmann por el concepto de intersubjetividad se remonta hasta el comienzo de su carrera académica y lo acompañó en el trascurso de esta. En su elaboración conceptual sobre la materia reconocemos dos grandes momentos. El primero de ellos está cristalizado en el primer volumen de Ilustración sociológica y lo situamos hacia finales de los años 60. El segundo momento fue inaugurado por Sistemas sociales y lo ubicamos hacia mediados de los años 80 , cuando el autor transformó el fundamento operativo de su programa de investigación. ${ }^{3}$ La divergencia entre ambos momentos es

\footnotetext{
Seguimos el concepto de fundamento operativo de Mascareńo (2008), quien, en diálogo con el programa de epistemología estructuralista de Wolfgang Stegmüller (1976) y con las innovaciones de Agustín Ibáńez (2006), lo define como articulación dinámica que permite a la teoría sintetizar sus componentes y ponerlos en funcionamiento.
} 
marcada: mientras el primero supone una consideración positiva del concepto, el segundo supone una fuerte crítica y una reformulación limitada. Para establecer su evolución, esquematizamos y cotejamos ambos momentos.

En el primer momento, Luhmann estrechó el concepto de intersubjetividad con los fundamentos operativos de su programa. En sus escritos programáticos iniciales, compilados en el primer volumen de Ilustración sociológica, el autor manifestó su pretensión de radicalizar el programa sistémico de Parsons a partir del reemplazo del concepto de sistema abierto por el concepto de sistema complejo y de la inversión en la prevalencia de la estructura y la función a favor de esta última. Guiado por estas pretensiones, el joven Luhmann reclamó una renovación en la construcción del objeto sociológico que incorpore el concepto de intersubjetividad. Según sus palabras: "Si la sociología desea lograr una representación (Vorstellung) de su problema de referencia en materia de análisis funcionales, la complejidad social deberá echar mano (zurückgreifen) a una teoría trascendental de la constitución intersubjetiva del sentido" (1973, p. 108).

Para satisfacer su propio reclamo, Luhmann elaboró una articulación original entre la teoría de la complejidad y las fenomenologías husserliana tardía y posthusserliana. Su eje fue el concepto de sentido social, el que definió como sentido intersubjetivamente constituido, es decir, basado en la coexperiencia y la formación de expectativas respecto del otro y dotado de una organización compleja. El concepto de sentido social, esto es, el concepto de intersubjetividad, designó el modo social de constitución de sentido, un orden de realidad específico dentro del mundo, dotado de un tipo de contingencia propio: la doble contingencia (Luhmann, 1973).

Luhmann fortaleció la posición de la intersubjetividad al ampliar su red de relaciones con los dos elementos básicos del primer fundamento operativo de su programa: el sistema y la acción sociales. Por un lado, el autor incorporó la intersubjetividad en la definición de sistema social, el que concibió como un sistema de acción estructurado por expectativas intersubjetivas. Así, la estructura de expectativas intersubjetivas conforma un nivel de organización del sentido de la acción, y distingue al sistema social de otro tipo de formaciones sistémicas (Luhmann, 1973), pues posibilita que la selección de la acción dentro de un sistema social esté orientada por expectativas reflexivas recíprocas entre los actores (Luhmann, 1973). ${ }^{4}$

\footnotetext{
El autor también convirtió el concepto en una propiedad y lo asoció con distintos conceptos cuando era menester deslindar esos conceptos de la esfera psicológica y situarlos bajo una perspectiva sociológica, a saber, por supuesto, 'sentido intersubjetivo' (intersubjektiv Sinn), pero además 'afirmación intersubjetiva' (intersubjektiv Aussage), 'transmisibilidad intersubjetiva' (intersubjektiv Übertragbarkeit). Mención aparte merece el concepto de 'certeza intersubjetiva' (intersubjektiv Gewißheit) -de innegable resonancia fenomenológica-, que Luhmann empleó a menudo en el primer volumen de Ilustración sociológica y sobre el que
} 
Por otro lado, el autor la vinculó con el concepto de acción social, al que dotó de unidad social, pues la acción social, en cuanto acción dentro de un sistema social, es presentada como una selección bajo un horizonte egológicamente dislocado de posibilidades abierto por, y sólo por, la experiencia del otro.

En ese primer momento, entonces, Luhmann elaboró un concepto de intersubjetividad fuerte, lo relacionó con las definiciones de sistema y acción sociales, y así lo elevó al rango de elemento necesario de la red de relaciones del fundamento operativo de su programa. El autor mantuvo el concepto a lo largo de los años 70, aunque su fuerza y presencia decrecieron conforme avanzaba la década, como testimonian los volúmenes segundo y tercero de Ilustración sociológica. ${ }^{5}$ Concebido en estos términos, registramos su última aparición en "Vivencia y acción" de 1978, y a partir de entonces se inicia la transición hacia el segundo momento. ${ }^{6}$

El segundo momento presentó una crítica fuerte y una reformulación limitada del concepto de intersubjetividad. Durante el primer lustro de los años 80, Luhmann alteró el fundamento operativo de su programa. ${ }^{7}$ A partir de ello dejó de concebir el objeto sociológico como sistema de acción intersubjetivamente organizado, para concebirlo como sistema emergente cerrado operativamente en la comunicación. El nuevo fundamento operativo explica el desplazamiento del concepto de intersubjetividad de su antiguo lugar. Por un lado, la unidad del sistema dejó de ser la estructura y comenzó a ser la operación, de manera tal que la marcada impronta estructural de la intersubjetividad perdió pertinencia para determinar la unidad del sistema social; por otro lado, el elemento último del sistema social dejó de ser la acción y comenzó a serlo la comunicación, de manera tal

basó otras nociones como 'entendimiento intersubjetivo' (intersubjektiv Vernunft), 'saber intersubjetivo' (intersubjektiv Wissen), 'verdad intersubjetiva' (intersubjektiv Wahrheit). En nuestro corpus no hallamos registros posteriores a 1969 de este concepto.

5 En ocasión de su primera polémica con Habermas, Luhmann (1971) contrapuso su concepto de intersubjetividad a la concepción habermasiana de intersubjetividad como supuesto racional de la comunalidad consensual entre interactuantes capaces de habla y acción, pues dicho concepto, primero, retrotrae la discusión sobre la intersubjetividad a premisas pre-fenomenología trascendental en tanto asume que no todo sentido objetivo es constituido intersubjetivamente; segundo, es incompleto porque excluye del concepto a la divergencia; tercero, plantea un dualismo entre interacción y sistema.

6 En ese artículo Luhmann mencionó el sentido intersubjetivamente constituido como uno de los elementos distintivos del sistema social (2009b).

7 Dicha transformación se basó en la modificación de tres elementos básicos del programa sistémico: 1) un giro autopoiético, es decir, la complejización de la teoría de sistemas autorreferenciales a través de la incorporación de los conceptos de autopoiesis y de clausura operativa; 2) un giro comunicativo, es decir, el paso de una teoría centrada en la acción a otra centrada en la comunicación, y 3) la adopción del enfoque de la constitución mutualista de elementos para dar cuenta de la emergencia de sistemas sociales. 
que la intersubjetividad, que complementaba la acción, perdió coherencia respecto de la necesaria unidad social de la comunicación.

La crítica de la intersubjetividad desde los nuevos supuestos fue formulada en 1986 en el muy citado artículo "¿Intersubjetividad o comunicación?” (Luhmann, 1998b). Allí, Luhmann, en primer lugar, encuadró la intersubjetividad dentro de la tradición de la teoría del sujeto y señaló que en ese contexto estuvo históricamente referida a la tensión entre la conciencia, enaltecida como subiectus, y la dimensión social. En segundo lugar, el autor señaló que, ante ese problema, la intersubjetividad no alcanza a constituir un concepto, sino apenas una fórmula de compromiso, dado que bosqueja una unidad precaria entre ambos términos, basada en un forzamiento; por tanto, sólo desplaza la tensión, no la resuelve. Luhmann consideró insanablemente débil la fórmula de compromiso por tres razones: 1) su composición terminológica encierra una contradicción entre el prefijo ('inter-') y el nombre ('sujeto', subiectus). En tanto el prefijo impone una base ontológica múltiple y simétrica de unidades equivalentes e irreductibles, el nombre impone una base ontológica una y única, cuya propiedad primordial es ser fundamento necesario de sí. 2) Su articulación lógica conduce a un vacío conceptual, pues está basada en el concepto de relación, el cual es vago e impreciso para determinar el vínculo entre subiectus y alteridad. 3) Su referente lleva hacia la paradoja de la conciencia intersubjetiva, puesto que introduce en la conciencia un elemento que no es conciencia, y así la fuerza a indicar lo no indicado por ella. Finalmente, en tercer lugar, la suma de estas debilidades (la contradicción entre lo múltiple y lo uno, el vacío del concepto de relación y la paradoja de la conciencia intersubjetiva) atentan, según Luhmann, contra la capacidad de unidad del concepto y fuerzan a la teoría a declinar de su elección a la hora de determinar la unidad operativa de los sistemas sociales; respecto de la cual resultó favorecido el concepto de comunicación, entendido como síntesis emergente de tres selecciones y no como 'transmisión' entre-sujetos.

Observamos que la divergencia entre el primer y el segundo momento es significativa. Mientras el primer momento presenta un concepto fuerte de intersubjetividad, incorporado al fundamento operativo del programa, el segundo presenta un concepto subordinado, debilitado frente a la comunicación, desacoplado del fundamento operativo. Este esquema de momentos divergentes explica de manera suficiente el cambio de estatuto del concepto de intersubjetividad en Luhmann, sin embargo, sólo ofrece una explicación parcial sobre los cambios de la conceptualización. Ello se debe a que el autor no redujo la intersubjetividad a la fórmula de compromiso del intersujeto, sino que consideró que su referente puede ser abordado desde el nuevo fundamento operativo. Por ello afirmamos que el propio Luhmann 
inició la tarea de concebir la intersubjetividad desde la comunicación (y no a la inversa). De manera tal que, en ese mismo texto de 1986, a renglón seguido de la crítica contra la 'fórmula de compromiso', el autor delineó una reformulación de la intersubjetividad a partir de los supuestos de la teoría de sistemas sociales comunicativamente clausurados.

La reformulación tuvo dos operaciones. La primera de ellas consistió en la introducción de una especificación estructural del concepto, que dejó de referir las expectativas sobre el otro en general, para referir un tipo particular de expectativas: la estructura de bifurcación entre consenso y disenso. La segunda operación consistió en la recuperación de dos componentes que en el primer momento habían sido acoplados laxamente al concepto y que en el segundo fueron estrechados a su (re)definición. Nos referimos, por un lado, al problema del disenso y a la distinción primaria entre consenso y disenso y, por otro lado, al concepto de concordancia. Luhmann procuró así enriquecer la perspectiva sistémica de la intersubjetividad. Con esta consideración damos paso al deslindamiento categorial del concepto.

\section{Reformulación comunicativa de la categoría de intersubjetividad}

Como se sigue del apartado anterior, la redefinición de intersubjetividad basada en el fundamento operativo comunicativo supone tanto la crítica a la fórmula de compromiso del intersujeto, como el esfuerzo por especificar su referencia estructural. En ella encontramos tres ejes primordiales: la prevalencia estructural, la formación de concordancia, y la equivalencia de consenso y disenso.

En cuanto al primer eje, Luhmann puso en primer plano la prevalencia de la función estructural de la intersubjetividad: "La intersubjetividad es, en definitiva, una estructura de la bifurcación entre consenso y disenso, y en su concepto no hay, ante todo, punto de apoyo alguno que permita considerar uno de los dos caminos como mejor que el otro" (1998b, p. 36). La postulación de esta prevalencia es determinante en tanto conecta en general la función intersubjetiva de la comunicación con la condensación de expectativas que conforma el nivel estructural de los sistemas sociales. En particular, las estructuras intersubjetivas son las estructuras que se forman en torno al consenso y al disenso; estos constituyen los horizontes de la dimensión social del sentido de la comunicación (Luhmann, 1998a) y, por tanto, concierne de manera directa a las expectativas sociales que se forman sobre temas, tematizaciones y contribuciones. En cuanto estructura comunicativa, la intersubjetividad está sujeta, primero, a los procesos de morfogénesis de los sistemas sociales, que inhiben o desinhiben determinadas posibilidades de selección, 
en virtud de un reforzamiento selectivo distribuido en mecanismos evolutivos de variación, selección y estabilización de estructuras; segundo, a la estilización cognitiva o normativa de las expectativas que forma; tercero, a la reversibilidad del sentido en el tiempo.

Sobre la base de este encuadramiento, la definición sistémica de intersubjetividad invierte los planteos que la consideran un supuesto necesario de la comunicación. Ello surge de la desvinculación entre los conceptos de estructura y medio asumida por Luhmann. El primer concepto designa, como dijimos, una condensación de expectativas dentro de la recursividad de la comunicación; el segundo, la desimprobabilización de las improbabilidades de la comunicación (Luhmann, 1998a). Al vincular la intersubjetividad con el primer concepto, y no con el segundo, Luhmann asumió que ella puede formarse o no, pero en ningún caso se convertiría en un medio. Por tanto, lejos de ser una condición necesaria de la comunicación, la intersubjetividad es un producto de esta.

En cuanto al segundo eje, Luhmann postuló que la formación de estructuras intersubjetivas implica la formación de concordancia: "Los sujetos pueden concordar (übereinstimmen) en el consenso y en el disenso; o dicho de otra manera: la concordancia es tan exigible para el consenso como para el disenso" (1998b, p. 35). Este eje es la encrucijada de la definición, pues es un eje tan endeble como promisorio. Ocupa un lugar importante en ella y apunta una innovación de fondo sobre la teorización sociológica de la intersubjetividad, pero no recibe una conceptualización adecuada y luce un tanto incompleto. Para paliar estas debilidades, realizamos un esfuerzo reconstructivo adicional. Nos remontamos hasta 1971, cuando el autor definió el concepto de formación de expectativas intersubjetivas en un sentido amplio. En ella encontramos varios elementos que permiten precisar la concepción de concordancia del autor. En ese momento, en ocasión de delinear el concepto de un horizonte de alcance mundial de posibilidades de acción y experiencia, Luhmann caracterizó el nivel de formación de expectativas intersubjetivas con tres elementos: primero, una concordancia fáctica (faktische Übereinstimmung) del horizonte en el cual las expectativas se constituyen; segundo, una expectativa de congruencia de horizontes de expectativas (Erwartung der Übereinstimmung des Erwartungshorizontes) de otros con los propios, y, tercero, una expectativa de que los otros tengan, a su vez, la expectativa de que su propio horizonte sea idéntico con el de otros (Luhmann, 2009a).

Si bien es cierto que de estos elementos no se desprende ni una definición clara y distinta de la concordancia ni un esclarecimiento de su común pertinencia tanto para el consenso como para el disenso, encontramos en ellos, no obstante, el indicio de que la concordancia atañe a un tipo de ordenamiento de 
las expectativas, cuya característica distintiva es una expectativa de heterogeneidad baja entre ellas. ${ }^{8}$

De esta manera, si nos apoyamos en ella para reconstruir la concordancia comunicativa, las expectativas intersubjetivas se distinguen por esa propiedad; ellas se forman en la dimensión social de sentido como un horizonte de expectativas donde se expecta una heterogeneidad baja entre temas, tematizaciones, contribuciones y semas. Dicha heterogeneidad baja puede modularse positiva o negativamente. Se modula positivamente cuando la formación de concordancia ordena expectativas de heterogeneidad baja respecto de una expectabilidad orientada hacia la aceptación, y es entendida como consenso. En cambio, se modula negativamente cuando la formación de concordancia (y no en desmedro de ella) ordena expectativas de heterogeneidad baja respecto de una expectabilidad orientada hacia el rechazo. Debido a esta relación ortogonal de la intersubjetividad con la aceptación y el rechazo, cuando la concordancia se modula consensualmente no se convierte en un equivalente funcional de los medios de comunicación simbólicamente generalizados (MCSG), ${ }^{9}$ pues el consenso es generado por estructuras de baja heterogeneidad orientadas hacia la aceptación, que al no estar orientadas a desimprobabilizar el éxito, no conciernen a la aceptación en cuanto selección, sino en cuanto posibilidad; por extensión, tampoco son capaces de reingresar la selección en la distinción entre motivación y selección.

A partir de esta caracterización de los rendimientos sistémicos de la intersubjetividad, señalamos su congruencia con el primer eje y ampliamos, así, la prevalencia estructural de la intersubjetividad, pues Luhmann, al vincular la función de la intersubjetividad con la concordancia, fue en detrimento del vínculo de ella con

8 Concedemos que la definición de 1971 presenta dos dificultades: por un lado, se trata de una definición indirecta, y, por otro, en ella predomina el vínculo acción-expectativas del primer momento; sin embargo, entendemos que no es incompatible con el fundamento operativo comunicativo, pues no concierne a la operación, sino a la estructura, por tanto, no contradice forzosamente el fundamento operativo. Apelamos a ella porque no hallamos en nuestro corpus una definición más estricta de Übereinstimmung, y además porque, a pesar de las dificultades que acarrea, nos permite reconstruir algunas características específicas del objeto. Esperamos, no obstante, que el trabajo de recopilación y los estudios críticos del recientemente creado Luhmann-Archiv arrojen en el futuro más y mejores respuestas sobre el concepto de concordancia. Por otra parte, si seguimos la distinción de Koselleck (1993) entre palabra y concepto, observamos que Luhmann alternó entre ambos usos, con predominio del primero. Por último, no queremos dejar de señalar que la definición de 1971 guarda similitudes con la definición de la reciprocidad de perspectivas de Schutz (1974).

9 Los medios de comunicación simbólicamente generalizados son logros evolutivos de la comunicación orientados hacia la consecución de la comunicación porque incrementan las probabilidades de aceptación o éxito al transformar en probable el hecho improbable de que una selección de álter sea aceptada por ego. Tales medios son el poder (poder/derecho), la verdad científica, el dinero (dinero/propiedad), el amor, el arte, los valores. 
el entendimiento (Verständigung) defendido por la perspectiva pragmático-formal de la acción comunicativa, a la que criticó en sus versiones formal (Habermas) y trascendental (Apel). La intersubjetividad desde la perspectiva sistémica no está funcionalmente referida a la improbabilidad del entendimiento (Verständigung) entre los álter ego interactuantes, y por ello no tiene ligazón interna con el medio del lenguaje, el medio que desimprobabiliza el entendimiento en la constelación álter ego en la evolución de la comunicación. Por tanto, no es performada por ningún acto ilocutivo del lenguaje ni está relacionada con la formación de la constelación diádica entre los álter ego interactuantes, sino que condensa expectativas hacia un horizonte de consenso o de disenso.

En cuanto al tercer eje, Luhmann asumió la equivalencia de consenso y disenso:

Cualquiera sea aquello que el "inter" de las subjetividades pueda enunciar [...] en todo caso existen dos posibilidades: una positiva y otra negativa. La intersubjetividad puede traducirse en consenso o en disenso. A ninguna de las dos posibilidades puede negarse la "interidad" ("Interität"). (Luhmann, 1998a, p. 35)

Desde este punto de vista, no es menos intersubjetivo el disenso que el consenso (Luhmann, 1998b). Esto significa que el disenso y el consenso son modulaciones posibles de una estructura intersubjetiva, por lo que la modulación no determina la formación de concordancia comunicativa, sino el signo de esta. De esta manera, si la distribución de expectativas de un sistema social condensa un horizonte de expectativas con heterogeneidad baja hacia la positiva (consenso) o hacia la negativa (disenso), se habrá formado una estructura intersubjetiva dentro del nivel de formación de estructuras de ese sistema. ${ }^{10}$ En cuanto tales, las estructuras intersubjetivas no son privativas de un tipo de sistema social en particular, no son propias de los sistemas de interacción del 'mundo de la vida', sino que son propias de los sistemas sociales en general. De esta manera, pueden formarse tanto en los sistemas de la diferenciación de planos (interacción, organización, sociedad) como en los de la diferenciación de subsistemas (segmentos, centro/periferia, estratos, funciones).

10 Luhmann consideró desde temprano la distinción entre consenso y disenso. Así, la hallamos ya en 1968, cuando el autor procuró identificar el desplazamiento de problemas propio de cada dimensión del sentido, y asumió que en la dimensión social la complejidad del mundo es traducida como problema de disenso entre perspectivas incongruentes. Este planteo está presente incluso en Sistemas sociales (Luhmann, 1998a). Es cierto también que, en todos estos casos, el autor consideró que el tratamiento más apropiado de esa distinción se daría en el ámbito de investigaciones 'socio-psicológicas', de la psicología social o de la sociometría; sin embargo, también es cierto que con la redefinición comunicativa de la intersubjetividad, Luhmann no reiteró ese encuadramiento, sino que la consideró un objeto de la sociología. 
Sergio Pignuoli Ocampo

Luhmann asumió, además, que la distinción entre consenso y disenso es una bifurcación, es decir, es igualmente probable (equiprobable) que un sistema social forme una estructura intersubjetiva de consenso o de disenso. La distribución en uno u otro sentido depende antes de la casualidad que de la argumentación racional de los "buenos motivos" (Luhmann, 1998b, p. 36, n. 13). ${ }^{11}$

$\mathrm{Al}$ no estar unilateralmente orientada hacia el consenso, la intersubjetividad tampoco está unilateralmente orientada hacia la consecución de la comunicación. De esta manera, se afirma por otra vía que la intersubjetividad, ni como función ni como estructura comunicativa, (desim) probabiliza el éxito. Por lo tanto, primero, no es un equivalente funcional de los Medios de Comunicación Simbólicamente Generalizados (MCSG), por lo tanto, no es alternativo a ellos; segundo, tampoco es ajeno a estos, dado que en el horizonte de expectativas continuamente reorganizado de los sistemas funcionalmente diferenciados apoyados en un MCSG siempre es posible la formación de estructuras intersubjetivas, moduladas ya hacia el consenso ya hacia el disenso. En este sentido, el concepto sistémico de intersubjetividad descarta por incompletas las pretensiones consensualistas que la fórmula de compromiso del intersujeto imprimió al concepto.

Hasta aquí llega nuestra reconstrucción del aporte realizado por Luhmann; para avanzar a partir de él nos interesa indagar en lo siguiente: ¿`cómo es formada la concordancia en los sistemas sociales?, y ¿̨con qué otros elementos del proceso recursivo básico se vincula?

11 Si bien Luhmann no fue explícito al respecto, intuimos que el concepto de bifurcación invocado dialoga con las teorías de la bifurcación, por entonces inspiradas en la teoría de las estructuras disipativas de Ilya Prigogine, quien proponía el concepto de punto de bifurcación para indicar el momento en que un sistema ingresa en una fase de no-equilibrio en pos de nuevos ordenamientos más complejos de autoorganización; una particularidad de esos puntos es que las diversas vías de complejización (bifurcaciones) que puede seguir para alcanzar ese nuevo ordenamiento son equiprobables y la selección de una de ellas en detrimento de otras igualmente probables es casual. Una presentación sintética de esta teoría se encuentra en Prigogine y Srengers (2004). Para reforzar esta intuición recordamos además que Luhmann seńaló que sus planteos sobre la estructura podían considerarse paralelos a los avances de ese programa (1998a). Dentro de esa línea de elaboración conceptual, algunos años más tarde, en el manuscrito de "Organización y decisión”, que se publicara póstumamente, Luhmann (2010) incorporó la noción de equifinalidad al concepto de consenso. De manera tal que, desde el punto de vista de la formación de consenso, los estados de las diversas conciencias individuales son tratados a los fines de la consecución de la comunicación como si todos tuvieran el mismo sentido (gleichsinnig). 


\section{La intersubjetividad como selección agregativa de sistemas sociales}

En el apartado precedente reconstruimos el concepto de intersubjetividad y precisamos una propiedad de su rendimiento: la formación de concordancia, que es una estructura de expectativas con una distribución de baja heterogeneidad de las expectativas en el horizonte de la dimensión social de sentido. En este apartado avanzaremos una hipótesis acerca de cómo se lograría tal distribución. Al respecto sugerimos que la concordancia se forma por agregación.

Esta hipótesis pone en línea la discusión acerca de la intersubjetividad con un intrincado aspecto de la teoría general de sistemas, a saber, el fenómeno de la agregación en sistemas complejos: ¿cómo es posible el fenómeno de la agregación en sistemas complejos? El enfoque de la cuestión de la agregación nos permite subrayar que estamos situando la intersubjetividad dentro de otro problema general poco explorado aún por el programa luhmanniano.

El propio Luhmann colaboró con esa escasa exploración, en tanto tendió a marginarla antes que a encontrarla productiva. Es posible que ello se deba a su consideración del concepto en el marco de su recepción de la teoría de la emergencia, donde, como sugiere William Wimsatt (1997), las ciencias composicionales suelen considerar la agregación y la emergencia como mecanismos alternativos. Luhmann siguió un criterio de este tipo y contrapuso agregación y selección. En detrimento de la primera entendió a la segunda como mecanismo no lineal, compatible con la emergencia; el mecanismo de agregación indicaría, en cambio, un hipotético mecanismo lineal de acumulación de complejidad intersistémica, pasible de ser formalizado como progresión aritmética e incapaz de dar cuenta de la emergencia y diferenciación de sistemas complejos. ${ }^{12}$

Esta contraposición entraña dos presunciones sobre la agregación. Primero, vuelve recíprocamente excluyentes las categorías y no considera la agregación como selección; segundo, limita la noción de agregación a ser una alternativa ontológica de la emergencia. La crítica que se sigue de ellas se vuelve improcedente ante una visión que no considere ni la agregación como mecanismo intersistémico, sino como mecanismo intrasistémico, ni como hipótesis ontológica sobre la formación de sistemas, sino como hipótesis de formación estructural de sistemas emergentes. Tal es la posición de algunos de los teóricos de sistemas citados por el propio

12 Reconstruimos este concepto a partir de los términos con que Sistemas sociales sostiene su posición respecto de la diferenciación operativa entre órdenes de realidad en el debate contra las posiciones de Edgar Morin y del bioconstructivismo cognitivo de Maturana y Varela (Luhmann, 1998a). 
Luhmann, como Magoroh Maruyama (1978), pero también es asumida por otras autoridades como el ya referido William Wimsatt.

Según esta perspectiva, ni la cuestión de la agregación se restringe a la discusión emergentista ni la crítica de la agregación intersistémica es extensiva a la agregación intrasistémica. La agregación es, más bien, un mecanismo de la formación de estructuras. Por ello nos inclinamos a vincular el concepto sistémico de intersubjetividad con esta visión estructural de la agregación, pues favorece una observación del proceso de aglomerado en la formación estructural. Desde ese punto de vista, el fenómeno de la agregación implica selección, ya que el aglomerado sólo puede formarse si la agregación se da como actualización de relaciones bajo un horizonte de otras posibilidades relacionales. Por esta razón, Wimsatt (1997) señaló que el fenómeno de la agregación no es homólogo al fenómeno de la aditividad, en tanto esta última supone una progresión de todas las propiedades de un sistema, e incluso de propiedades ajenas al sistema.

Asimismo, el concepto de autorreforzamiento de Maruyama nos ayuda a esclarecer nuestro desarrollo, pues, según el epistemólogo japonés, el autorreforzamiento de elementos describe la amplificación selectiva de un elemento como un proceso de feed-back positivo, es decir, procesos que tienden a incrementar la información y a generar estados organizados de un nuevo orden mediante la interacción y la desviación. La agregación también es un mecanismo selectivo de amplificación, pero no de elementos, sino de relaciones. La agregación autorrefuerza relaciones de baja heterogeneidad, pero, lejos de lo que podría creerse, ello no conduce al sistema ni hacia un proceso de homogeneización ni hacia uno de simplificación, sino que, por el contrario, incrementa la heterogeneidad estructural de este, dado que la agregación supone procesos de causación recíproca para lograr la amplificación. ${ }^{13}$ En consecuencia, cuando los procesos de autoorganización involucran procesos de agregación, conducen al sistema hacia estados de mayor complejidad y de mayor información en el nivel de sus estructuras; los cuales, a su vez, corren en paralelo con los procesos de autorreforzamiento de elementos, por lo que pueden disparar a su vez otros procesos de desviación amplificante y causación recíproca.

En rigor, si bien Luhmann, como dijimos, no abogó por la agregación en el nivel de la formación de sistemas, asumió, no obstante, dos equivalentes funcionales en el nivel de la formación de estructuras: la condensación y el

13 La distinción entre epistemologías homogeneista y heterogeneista promovida por Maruyama (1978) es relevante aquí, pues ni siquiera los procesos de agregación imponen una interpretación homogeneista. 
autorreforzamiento de la variación. ${ }^{14}$ El primero da cuenta de la formación de redundancia en virtud de la iteración de una indicación. El segundo introduce un elemento dinámico en la distinción entre los mecanismos evolutivos de variación y selección, y ofrece una descripción del modo en que un elemento desviante puede disparar un proceso de feed-back positivo al nivel de las estructuras. Desde la perspectiva que mencionamos arriba, ambos pueden aunarse en el concepto intrasistémico de agregación y así ser traspuestos en un esquema de generalidad mayor: la condensación agrega redundancia y el autorreforzamiento de la variación agrega elementos desviantes. Entendemos ambos como casos de agregación en los sistemas sociales.

A partir de estas discusiones alcanzamos una definición de agregación, a la que entendemos como mecanismo intrasistémico de formación de estructuras a partir de la amplificación selectiva de determinadas relaciones entre las relaciones de ese sistema. La agregación amplifica una premisa organizativa dentro del sistema, el rasgo distintivo de este tipo de amplificación es el rango de dispersión bajo (varianza) de aquella premisa.

El concepto de intersubjetividad que reconstruimos en el apartado precedente es compatible con ella. La concordancia se forma por agregación, desde que amplifica determinadas expectativas comunicativas con determinada modulación entre otras expectativas posibles y disminuye el rango de dispersión en torno a ellas. Desde el punto de vista del concepto de agregación, en los casos en que se produjera esta amplificación ambas modulaciones de la intersubjetividad equivalen, pues es indistinto si una agregación se modula como consenso o como disenso.

De esta manera, a partir de la definición de agregación, se refuerza la idea de que las estructuras intersubjetivas no están orientadas hacia la tercera improbabilidad de la comunicación, ya que, en virtud de su amplificación, son ortogonales -es decir, duplican los grados de libertad operativa- respecto de la distinción entre aceptación y rechazo; de esta manera generan rangos de expectabilidad, eventualmente simultáneos si una tematización genera en paralelo consenso y disenso, para la aceptación y/o el rechazo. Por esta razón, la intersubjetividad comunicativa no queda referida ni a estructuras generales ni a la formación de un sentido común, sino, más bien, a estructuras específicas de sentidos, compatibles tanto con una autoorganización policéntrica y, even-

14 Un tercer equivalente podría ser el concepto de cristalización (Herauskristallizierung, Ankristallisierung), que Luhmann asumió tempranamente, y que nunca abandonó, aunque tampoco ponderó lo suficiente. 
tualmente, con una coordinación y direccionamiento en disputas, como con una autoorganización concéntrica. Finalmente, la intersubjetividad, en cuanto estructura de expectativa, es temáticamente reflexiva, razón por la cual siempre es alterable e incluso desorganizable, al ser tematizable la concordancia respecto del nivel de consenso o disenso logrados. Ello genera aperturas para nuevas formaciones de intersubjetividad, incluso en un nivel de segundo orden si se forma una estructura de expectativas concordante (hacia el consenso o hacia el disenso) respecto de otra estructura de expectativas concordante (hacia el consenso o hacia el disenso).

\section{La conexión entre intersubjetividad y la distinción entre temas y contribuciones}

Para completar nuestro argumento entroncaremos el concepto de intersubjetividad con el proceso comunicativo básico. De esta manera, lo integraremos dentro del sistema de categorías relativo a la recursividad operativa de los sistemas sociales, e introduciremos en este algunas distinciones adicionales. La vía más firme que encontramos para llevar esto a cabo fue conectar la intersubjetividad con la distinción procesal básica de la comunicación: la distinción entre temas y contribuciones.

Luhmann (1998a) afirmó que, gracias a la distinción entre temas y contribuciones, la comunicación se convierte en proceso y estiliza su organización. Los temas son definidos como estructuras de expectativas que organizan el sentido de la comunicación y que funcionan por generalización: "Los temas sirven como estructuras (Strukturen) concreto-temporal-sociales del proceso comunicacional y fungen en él como generalizaciones en la medida en que no determinan cuáles aportaciones serán otorgadas, ni cuándo, en qué orden y por quién" (1998, p. 156; trad. modificada).

Las contribuciones, en tanto, son definidas como aportes individuales orientados hacia y ordenados por un tema. La distinción entre tema y contribución, primero, establece una diferencia de niveles entre ambos: las contribuciones son coordinadas y ordenadas por los temas; segundo, declara la aceptación del tema, condición previa para la negación, corrección o modificación de contribuciones; tercero, dispone un horizonte de barreras temáticas; cuarto, abre en la dimensión social un horizonte del contenido expectable sobre las contribuciones (Luhmann, 1998a).

En este último aspecto, la dimensión social de los temas, encontramos la apertura que nos permitirá, primero, distinguir tema y tematización; segundo, vincular 
la intersubjetividad con esta última. Para ello necesitamos indagar con mayor detalle en la dimensión social de los temas. Notamos así que el acento puesto por Luhmann sobre el carácter generalizado de los temas produce una ambigüedad respecto de las estructuras de los sistemas sociales.

Esta ambigüedad se manifiesta en la propiedad de 'contenido de exigencia excesiva' (Zumutungsgehalt). Luhmann (1998a) refiere con este concepto la determinación de límites para la participación en la comunicación a partir de la expectabilidad del contenido de las contribuciones. En este sentido, la propiedad de contenido de exigencia excesiva designa la regulación de los límites sistema/ entorno. Según sus palabras:

La limitación se remite, por fin, a un proceso de negociación (la anticipación silenciosa, escondida o abierta); pasa por la autosimplificación del sistema, y así la acción de comunicación es tolerada o no en el sistema. Este proceso se deja dirigir a través de expectativas temáticas (Themenerwartungen). En los temas aceptables se dejan leer los límites del sistema. Junto a los temas/límites-determinados directos existen también los indirectos. Junto a la dimensión objetiva también las dimensiones temporal y social ofrecen posibilidades de regulación de límites [...]. Por medio de la dimensión social se deja regular, finalmente, aquello que debe considerarse como acción en el sistema y qué acciones son atribuibles al entorno. Con ello, los límites del sistema adquieren una precisión adicional atribuible a la autodescripción del sistema como sistema de acción. (Luhmann, 1998a, pp. 188-189)

La ambigüedad surge de las fuentes que Luhmann reconoce para la determinación de la 'exigencia excesiva'; en sus palabras:

La exigencia excesiva (Zumutung) de los temas, [que] altera los límites del sistema, se deja dirigir a través de la historia anterior (Vorgeschichte), a través de aquello que en esa situación es precisamente posible; pero también a través de las estructuras de expectativas generales. Estas estructuras pueden prever cómo y acerca de qué se comunica en el supermercado, en la radio, en la parada del tranvía, en la comida familiar, en la solicitud telefónica de un boleto de avión, etcétera. (Luhmann, 1998a, p. 189)

Luhmann reconoce dos fuentes: la recursividad de la situación y las expectativas generales. La ambigüedad surge del hecho de que la primera es propia de la 
autoorganización del sistema social; la segunda, en cambio, es exterior a dicha autoorganización, es proveída por las expectativas generales. Al aunarlas, la referencia de las estructuras temáticas se vuelve ambigua.

Esa ambigüedad, puesta de manifiesto por la propiedad de contenido de exigencia excesiva, está presente en toda estructuración temática de un sistema social que posea tanto una historia anterior como expectativas generalizadas. De ahí que nos invita a concebir este nivel estructural como un multinivel o nivel multiestratificado, que requiere distinciones ulteriores para, al menos, deslindar las expectativas generalizadas, ancladas en las estructuras semánticas de nivel societal, de las expectativas temáticas que forma un sistema social específico. Aquí nos inclinamos a denominarla distinción entre tema y tematización. Si el tema refiere las ya mencionadas expectativas semánticas generales, la tematización refiere la selección semántica que opera un sistema social al diferenciar entre temas y contribuciones. La semántica de la tematización ni es lineal ni se corresponde punto por punto con las semánticas generalizadas; se trata más bien de una selección a nivel estructural, que se forma a través de la recursividad de los aportes, y no se deduce de las estructuras generalizadas del tema. Su estructuración es selectiva, ya que no es actualizada la totalidad de generalizaciones y expectativas de un tema, por lo que la tematización constituye una reducción de complejidad semántica operada por un sistema social específico, el que, en virtud de la complejidad semántica de los temas, selecciona una tematización y estiliza sus propias expectativas sobre las contribuciones.

La propiedad agregativa de la intersubjetividad nos indica que la concordancia únicamente puede formarse a través de la amplificación de estructuras del propio sistema, y no de la amplificación de estructuras generales, debido a que la agregación no es aditiva, sino selectiva, incluso a nivel semántico. Por consiguiente, no hay estructuras intersubjetivas de las estructuras generales de los temas, sino estructuras intersubjetivas en torno a las tematizaciones.

De esta manera, aclaramos la conexión de la intersubjetividad con el proceso comunicativo y puntualizamos los factores a los que se vincula primariamente. La intersubjetividad se forma en la tematización antes que en el tema y conforma su dinámica de amplificación a través de la recursividad. En ella forma un horizonte de expectativas de baja heterogeneidad entre las expectativas de tematización, modulado por consenso y/o disenso, y es capaz entonces de coordinar las contribuciones e introducir diferencias entre ellas en un nivel distinto del nivel temático. Al mismo tiempo establece una diferencia de niveles entre su propio nivel de expectativas de baja heterogeneidad y las contribuciones expectables. ${ }^{15}$

15 También se abre el campo de la teoría de los medios de comunicación simbólicamente generalizados para 


\section{Conclusiones}

A lo largo de este trabajo hemos deslindado un concepto de intersubjetividad desde la perspectiva de la teoría general de sistemas sociales. Los pasos dados fueron diversos. Por un lado, reconstruimos la reformulación que el propio Luhmann dio al concepto; por otro, a partir de ella, avanzamos una fundamentación de la intersubjetividad basada en el concepto de agregación en sistemas complejos, y finalmente entroncamos el concepto resultante con el proceso comunicativo básico, a través de la distinción tema/tematización.

En cuanto a la reformulación luhmanniana, según nuestra reconstrucción, tras su crítica a la fórmula de compromiso del intersujeto, el autor dio al concepto nuevos lineamientos, acordes con el fundamento operativo comunicativo de su programa. Para ello hizo hincapié en tres ejes: la prevalencia estructural, la formación de concordancia y la equivalencia de consenso y disenso.

En cuanto a la vinculación de la intersubjetividad con el fenómeno de la agregación, identificamos la dimensión intrasistémica del fenómeno y procuramos aunar en él los diversos, aunque inconexos, equivalentes funcionales planteados por Luhmann. De esta manera logramos caracterizar estructuralmente la intersubjetividad como una formación específica de estructuras de expectativas de baja heterogeneidad.

En cuanto a su entroncamiento con el proceso comunicativo básico, indagamos en la distinción entre temas y contribuciones, introdujimos una diferenciación ulterior entre tema y tematización, y logramos asociar la intersubjetividad con esta última; así vinculamos su formación con las condiciones de posibilidad de la recursividad comunicativa del sistema social.

Por estas distintas vías entendemos que la intersubjetividad se caracteriza por agregar concordancia comunicativa y por modularla, bien como consenso bien como disenso. Esta concordancia es una estructura específica, caracterizada por un horizonte de expectativas de baja heterogeneidad formada en torno a una tematización y a través de la recursividad de un sistema social, lo que es congruente con nuestra hipótesis inicial. Asimismo, en cuanto estructura específica, la intersubjetividad es compatible con todos los niveles de la diferenciación de planos (interacciones, organizaciones, sociedades) y de la diferenciación de subsistemas (segmentos, centro-periferias, estratos, funciones).

la categoría de intersubjetividad, ya que la formación de concordancia puede darse a través de la codificación de expectativas y fortalecer o debilitar un nivel intermedio de expectativas de tematización, diferenciados respecto de la generalización simbólica de los distintos medios de comunicación tecnificados. 
Estos resultados nos llevan a ponderar nuestro concepto de intersubjetividad desde el punto de vista del dilema teórico entre comunicación e intersubjetividad. Puesto en esa perspectiva, nuestro concepto indica una conexión interna entre intersubjetividad y comunicación, y caracteriza dicha conexión como una relación de dependencia de la primera con respecto a la segunda, debido a que la intersubjetividad es concebida como un tipo específico de estructuración comunicativa de expectativas. En ello reside nuestro aporte a la discusión de aquel dilema: la reformulación comunicativa de la intersubjetividad cuestiona la contraposición teórica y/o metodológica forzosa entre intersubjetividad y comunicación, y propone, en cambio, una rearticulación conceptual básica entre ambos conceptos. Una de las ventajas principales de este aporte es la apertura amplia e integrada que propone para el desarrollo de nuevas hipótesis de investigación, tanto teóricas como aplicadas, sobre temas vedados por aquel dilema.

En materia de investigación teórica abre un promisorio panorama para profundizar la indagación conceptual en todos los niveles de la teoría de sistemas sociales. A modo de ejemplo, señalamos dos de esos niveles. Al nivel de la teoría general de sistemas sociales hace foco sobre la conexión entre tematización semántica y formación de estructura de los sistemas sociales y sobre los diversos niveles de tensión estructural de estos sistemas. En tanto que al nivel de la teoría de la sociedad abre un tema vedado por el dilema intersubjetividad/comunicación, a saber, la conceptualización de la formación de intersubjetividad de los sistemas funcionalmente diferenciados.

En materia de investigación aplicada, el concepto de intersubjetividad abre un panorama amplio para la investigación empírica de las condiciones, la formación y la dinámica de la concordancia. En calidad de hipótesis para un futuro trabajo ofrecemos un ejemplo analítico sobre ello. Nos referimos a las redes sociales del medio de comunicación digital. Desde el programa sistémico, las redes sociales son observadas como una adquisición evolutiva notable, debido a que han posibilitado y robustecido la comunicación hacia el exterior, la 'comunicación hacia fuera' (Nach-Außen Kommunikation) de las organizaciones, pero fundamentalmente de las personas. ${ }^{16}$ Ello hace de las redes sociales un buen ámbito

16 Recordemos que según Luhmann (2007) las organizaciones eran el único plano sistémico que, en la modernidad, habían alcanzado y consolidado la comunicación hacia el exterior. La 'comunicación hacia afuera' de las personas es, sin dudas, una adquisición evolutiva de las redes sociales. Esta adquisición combinada con el alcance global del medio de comunicación digital convierte a las redes sociales en una auténtica innovación estructural de la sociedad mundial, en el sentido de las Eigen-Structures de Rudolph Stichweh (2012). En este punto, la indagación empírica de las redes sociales y la intersubjetividad se abre a los aportes sistemáticos sobre la diferenciación funcional en América Latina según fueran desarrollados por Das Neves (1994), Mascareño (2000), Chernilo y Mascareño (2005) y Calise (2012), entre otros. 
para desarrollar investigación empírica sobre la dinámica comunicativa de la intersubjetividad. En ellas, la formación y transformación de la concordancia es permanente. Esto nos permite indagar con mayor profundidad en el carácter eventual y precario de la formación comunicativa de concordancia, y observar la relación entre dichas características y el proceso recursivo básico de los sistemas sociales. Al mismo tiempo, su apertura y su flexibilidad temáticas nos permiten distinguir entre tema y tematización, y reconstruir a partir de ella la diversidad de disputas, e incluso la diversidad de estructuras intersubjetivas que se amplifica sobre la base de la formación múltiple de concordancias. Asimismo, no cabe despreciar la riqueza y la precisión de los datos que nos permitirían construir los diversos indicadores de conectividad. ${ }^{17}$ De esta manera, tanto por la vía del ejemplo analítico como de las potenciales indagaciones teóricas, destacamos la apertura y la plausibilidad investigativas que genera el paso del dilema intersubjetividad/comunicación al concepto comunicativo de intersubjetividad.

Para concluir este trabajo nos proponemos conectar estos resultados con dos líneas de investigación actuales: la propuesta de Urs Stäheli, por un lado, y la vinculación entre la Teoría General de Sistemas Sociales y análisis conversacional, por otro.

El trabajo de Stäheli (2000) planteó una sólida unificación de los análisis de la comunicación, la semántica y el discurso desde la perspectiva sistémica. En términos generales, este autor ha elaborado sus objetos de investigación en el nivel del análisis societal y en el nivel del análisis político. La tesis que recorre sus diversos estudios es que la semántica posee una 'estructuralidad' específica, pero no separada de la estructuración del sistema societal. El concepto de intersubjetividad que hemos alcanzado le ofrece a dicha perspectiva un nivel de desagregación, primero, porque revincula semántica y estructura, aunque al nivel del proceso recursivo, por tanto, abre esa dimensión de análisis para toda formación sistémica, y ya no sólo para el nivel societal $;{ }^{18}$ segundo, porque delinea una primera aproximación a las tensiones semánticas y sus distintos niveles (tema/tematización) en todos los

Agradezco la sugerencia de uno de los evaluadores anónimos de incorporar en las conclusiones un ejemplo analítico, que muestre la plausibilidad del concepto de intersubjetividad de cara a investigaciones futuras. Debo señalar, además, que el ejemplo que he ofrecido supone y se enmarca dentro de los resultados alcanzados por el proyecto de investigación en curso sobre las Tecnologías de la Información y la Comunicación desde la perspectiva de la Teoría General de Sistemas Sociales (Forte et al., 2012; Pignuoli Ocampo, 2012).

18 Recordemos que, si bien Luhmann (1998a) no negó la vinculación entre semántica y estructura en los sistemas sociales, no la consideró relevante para establecer la propiedad de coordinación de las estructuras respecto de la acción. Stäheli (2000) abogó por la revinculación a partir de rechazar la declaración de irrelevancia y destacar la relación entre semántica y acción, observable en el rol de la semántica en la autosimplificación del sistema comunicativo como sistema de acción, atribuido por el propio Luhmann (1998a). 
sistemas sociales, lo cual, a su vez, permitiría retomar y revisar la diferenciación de planos, incluyendo la dimensión semántica.

La línea de investigación que procura conectar la Teoría General de Sistemas Sociales con el análisis conversacional tiene una historia mayor, que podemos rastrear en el interés del propio Luhmann por la pragmática interpersonal de la conversación de Watzlawick y luego por la teoría de la conversación de Pask. Esta perspectiva procura combinar el análisis sistémico de la comunicación y de la doble contingencia con el análisis conversacional de la interacción. Baraldi y Gavioli (2007) vincularon ambas perspectivas por la común concepción medial del lenguaje. Reich (2010) propuso vincular la doble contingencia de Luhmann con la etnometodología de las situaciones de Garfinkel y con el análisis del lenguaje conversacional y de la toma de turnos de Schlegoff. El concepto de intersubjetividad que hemos deslindando ofrece a esta línea de investigación la posibilidad de desagregar niveles de estructuración y tensionamiento en la propia interacción. Consideramos factible, además, una segunda conexión, más ambiciosa, pues esta línea aborda con seriedad y radicalidad el análisis de la interacción en los subsistemas funcionales. En este sentido, el concepto de intersubjetividad ofrece las mencionadas prestaciones en materia de desagregación, y además cuestiona toda dualización entre sistemas funcionales y sistemas interaccionales basada en la intersubjetividad, desde que esta ya no es considerada monopolio de los últimos, sino una estructura formable en cualquier plano de la diferenciación sistémica. Con esta última consideración concluimos este trabajo, pues ella justifica plenamente la exploración que hemos emprendido en pos de establecer un concepto luhmanniano de intersubjetividad.

Recibido agosto 21, 2013 Aceptado diciembre 13, 2013

\section{Referencias bibliográficas}

Baraldi, C., Gavioli, L. (2007). The relevance of interactions in functionally differentiated society: The contribution of conversation analysis to the theory of social systems. Soziale Systeme $13(1+2), 125-135$.

Calise, S. (2012). El lugar del derecho en la diferenciación funcional: dos modelos latinoamericanos. Persona y Sociedad XXVI (3), 109-137.

Chernilo,D., Mascareño, A. (2005). Universalismo, particularismo y sociedad mundial: obstáculos y perspectivas de la sociología en América Latina. Persona y SociedadXIX (3), 17-45.

Forte, M. Á., Pignuoli Ocampo, S., Calise, S., Palacios, M., Zitello, M. (2012). Las TIC como problemática de la teoría sociológica. Entramados y perspectivas 2 (2), 205-226. 
Ibáñez, A. (2006). Complejidad y cognición. Thesis (PhD), Departamento de Psicología, Universidad Católica de Chile. [Tomo la referencia de Mascareño, A. (2008). Acción, estructura y emergencia en la teoría sociológica. Revista de Sociología 22, 217-256.]

Koselleck, R. (1993). Futuro pasado. Para una semántica de los tiempos históricos. Barcelona: Paidós.

Luhmann, N. (1971). Systemtheoretische Argumentationen. Eine Entgegnung auf Jürgen Habermas. En N. Luhmann y J. Habermas, Theorie der Gesellschaft oder Sozialtechnologie? (pp. 291-405). Frankfurt a.M.: Suhrkamp. (1973). Ilustración sociológica y otros ensayos. Buenos Aires: SUR. (1998a). Sistemas sociales. Lineamientos para una teoría general. Barcelona: Anthropos. (1998b). ¿Intersubjetividad o comunicación? Dos diferentes puntos de partida para la construcción de una teoría sociológica. En N. Luhmann, Contingencia y modernidad. De la unidad a la diferencia (pp. 31-50). Madrid: Trotta. (2007). La sociedad de la sociedad. México D.F.: Herder. (2009a). Die Weltgesellschaft. En N. Luhmann, Soziologische Aufklärung 2. Aufätze zur Theorie der Gesellschaft (pp. 63-88). Wiesbaden: VS Verlag. (2009b). Erleben und Handeln. En Niklas Luhmann, Soziologische Aufklärung 3. Soziales System, Gesellschaft, Organisation (pp. 77-92). Wiesbaden: VS Verlag. (2010). Organización y decisión. México D.F.: Herder.

Maruyama, M. (1978). Heterogenistics and morphogenetics: Toward a new concept of the scientific. Theory and Society 5 (1), 75-96.

Mascareño, A. (2000). Diferenciación funcional en América Latina: los contornos de una sociedad concéntrica y los dilemas de su transformación. Persona y SociedadXIV (1), 187-207. (2008). Acción, estructura y emergencia en la teoría sociológica. Revista de Sociología $22,217-256$.

Neves, M., das (1994). A Constitucionalizaçao Simbólica. San Pablo: Editora Académica.

Pignuoli Ocampo, S. (2012). Digitalización y convergencia tecnológica desde el punto de vista sociológico de la Teoría General de Sistemas Sociales. Gestión de las Personas y la Tecnología 5 (13), 153-165.

Prigogine, I., Stengers, I. (2004). La nueva alianza. Metamorfosis de la ciencia. Madrid: Alianza.

Reich, W. (2010). Three problems of intersubjectivity - And one solution. Sociological Theory 28 (1), 40-63.

Schneider, W. (1994). Intersubjetivität als kommunikatives Konstruktion. En P. Fuchs y A. Göbel (comps.), Der Mensch — das Medium der Gesellschaft (pp. 189-238). Frankfurt a.M.: Suhrkamp.

Schutz, A. (1974). Escritos 1. Buenos Aires: Amorrortu.

Stäheli, U. (2000). Sinnzusammenbrüche. Göttingen: Velbrück Wissenschaft.

Stegmüller, W. (1976). The Structure and Dynamics of Theories. New York-Berlin, Springer. 
Stichweh, R. (2012). En torno a la génesis de la sociedad mundial: innovaciones y mecanismos. Revista MAD 26, 1-16.

Wimsatt, W. (1997). Aggregativity: Reductive heuristics for finding emergence. Philosophy of Science 64, Supplement. Proceedings of the 1996 Biennial Meetings of the Philosophy of Science Association. Part II: Symposia Papers, 372-384. 